\title{
Micro Climate Monitoring-Web Application using Wireless Sensor Network
}

\author{
Ramesh $\mathbf{G}^{1}$, Nivedha $\mathbf{R}^{2}$ \\ Dr N.K Kartikeyan is with the Department of Information Technology, Sri Krishna College, Coimbatore, India
}

\begin{abstract}
This paper provides a brief overview about Micro climate monitoring and how we monitor the climate into a remote system. The traditional method has a problem of poor accuracy and wastage of labor. So we need a system to provide interface for collecting and analyzing the measured values and to provide the collected data for processing and necessary control so as to continuously monitoring the system remotely over Internet. This project provides a comprehensive review of the available solutions to wireless sensor network environmental monitoring applications.
\end{abstract}

Keywords: Sensors, ZigBee, PIC Micro-controller, GSM Inter-face Kit, Windows Azure

\section{Introduction}

Environmental monitoring has a long history. In early times analog mechanisms were used to measure physical environmental parameters. Some of them with the ability to record the values on paper dish. The old mechanisms recorded data at specific intervals and required human intervention to download them. Some years ago, digital data loggers have replaced the old mechanical. The digital data loggers are more easy to operate and to maintain and cheaper than the old mechanisms. Digital data loggers may also be combined with long-range communication networks, such as GSM, to retrieve data from remote sites. However, digital data log-gers have some drawbacks. The digital data loggers solution, usually provide monitoring at one point only and in many cases multiple points need to be monitored. There is not a standard to store data and to communicate with the data logger, so several different solutions are used. Recent advances in micro-electromechanical systems and in low-power wireless network technology have created the technical conditions to build multi-functional tiny sensor devices, which can be used to observe and to react according to physical phenomena of their surrounding environment. Wireless sensor nodes are low-power devices equipped with processor, storage, a power supply, a transceiver, one or more sensors and, in some cases, with an actuator. Several types of sensors can be attached to wireless sensor nodes, such as chemical, optical, thermal and biological. These wireless sensor devices are small and they are cheaper than the regular sensor devices. The wireless sensor devices can automatically organize themselves to form an ad-hoc multi hop network. Wireless Sensor Systems (WSSs), may be comprised by hundreds or maybe thousands of ad-hoc sensor node devices, working together to accomplish a common task. Self-organizing, selfoptimizing and fault-tolerant are the main characteristics of this type of network

Widespread networks of inexpensive wireless sensor devices offer a substantial opportunity to monitor more accurately the surrounding physical phenomenas when compared to tradi-tional sensing methods. Wireless sensor network has its own design and resource constrains. Design constrains are related with the purpose and the characteristics of the installation envi-ronment. The environment determines the size of the network, the deployment method and the network topology. Resources constrains are imposed by the limited amount of energy, small communication range, low throughput and reduced storage and computing resources. Research efforts have been done to address the above constrains by introducing new design methodologies and creating or improve existing protocols and applications. It provides a review on Wireless Sensor Systems solutions to environmental monitoring applications. The remainder of this paper is organized as follows. Section II gives an overview of sensor network platforms. Section III Micro climate monitoring and the data collection while Section IV overviews our web application. RMMS climate monitoring projects are presented $\mathrm{V}$ concludes the paper and addresses future research challenges related to Wireless sensor networks deployment and what are the technology we may include in sensor network.

\section{Sensor Network}

In the old Sensor networks technology that gained momentum over the years and it is very promising in making the vision of Mark Weiser a reality: Conceive a new way of thinking about computers in the world, one that takes into account the natural human environment and allows computers themselves to vanish into the background. Fortunately for researchers, this new technology brings up a wealth of research problems that need to be solved. Such networks are consider-ably different from the traditional computer networks we have been building over the past years, and thus they may have different requirements and/or constraints in each of the seven OSI layers. Some of these problems are briefly described next. When reading research papers relating to sensor networks, overwhelmingly, there are two main issues that rightly get great attention and differentiate sensor networks from the networks we know and understand. These are the limited power and the potential of deploying networks with huge number of sensor nodes. Depending on the application, the power constraint is really critical. For example, as described in the next section, at the Great Duck Island sensors are installed during the offseason and it is important that there is no human intervention during breeding season (7-9 months). As a result, the nodes should conserve energy so that the network as a whole will perform its tasks over that period. 


\section{International Journal of Science and Research (IJSR) \\ ISSN (Online): 2319-7064}

Index Copernicus Value (2013): 6.14 | Impact Factor (2015): 6.391

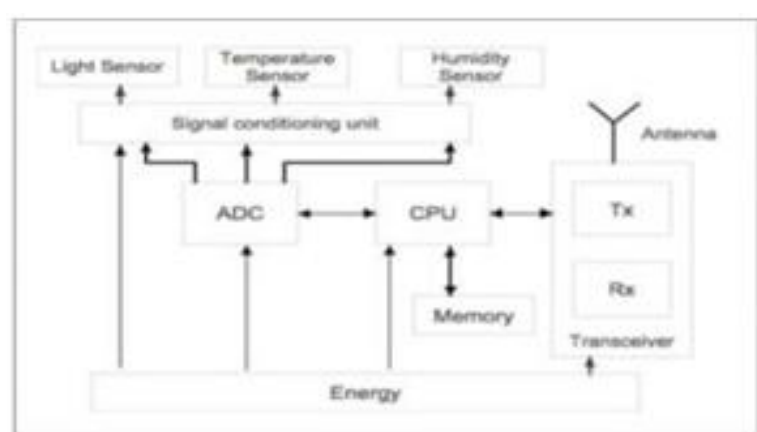

Figure 1: Sample Sensor Network Model

This is a significant constraint which affects the sensing, processing and communication capabilities of the sensor nodes as well as the protocols used to for coordination. The other im-portant issue associated with sensor networks, the huge number of sensor nodes that will be involved, is important because it is no longer possible to have a unique identifier for each sensor (e.g., an IP address). The addressing issue has implications to the media access control (MAC) protocols, routing protocols as well as reliable communication. Furthermore, the huge number of sensors has the potential of generating a vast amount of measurement data and therefore we need efficient algorithms for making sense out of the raw data, and turn them into useful information that humans can use. Measurements from sensor nodes capture the spatial and temporal state of the field. Thus, it is desirable to find ways of correlating the data and draw inferences that can improve the decision making process.

\section{Micro Climate Monitoring System}

The micro climate Monitoring process is implemented with the monitoring devices based on the wireless sensor system. Wireless sensor system consists of various sensor nodes to sense and gather data and the Base station to receive data from sensor nodes and then send the data to the system through ZIG-BEE. These devices adopt Temperature, Humidity and Soil moisture sensors are useful for micro climate monitoring system.

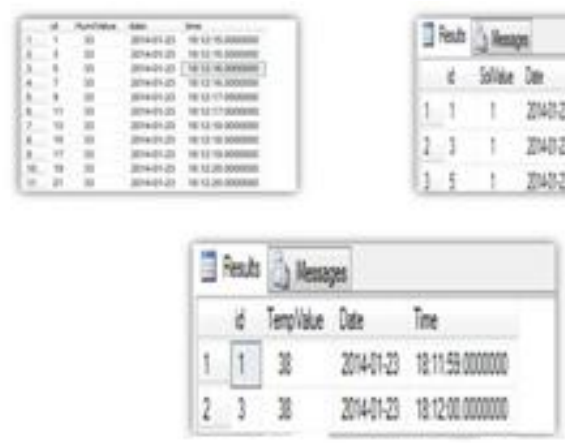

Figure 2: SQL-DB Table

These sensor nodes are sense environmental parameters such as Temperature, relative humidity and Soil moisture from their surrounding and send these parameters to the base station (ZIGBEE). The base station (ZIGBEE) connected to the host PC through which the proposed system displays the readings. The wireless sensor systems are deployed in the field. The sensor nodes sense the various parameters and send the data to the base station. The base station in turn sends the data to the live monitor view. The Live monitor interprets the data received from the base station. These data are displayed to the user. In the meantime, it stores the data in the database SQL. The drawback assist with this is that data do not accessed remotely.

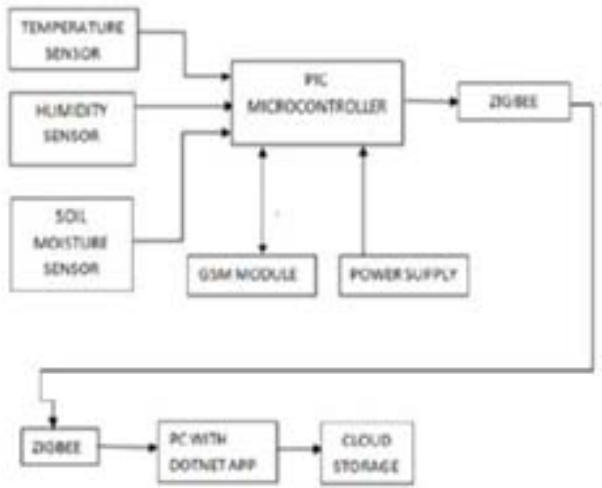

Figure 3: New Sensor Netowrk

The working of the monitoring system is classified into various modules and explained in the previous chapters. The working layout of MMS is depicted in the figure. After measuring the environmental parameters the user enters into the updating. Here user first login into the system. Then the system passes the query to the SQL database where all the measurements are stored and the SQL returns all the readings measures on the live values.

\section{Remote Monitoring}

The Remote Monitoring is interrelated with the Micro climate Monitoring system. In the MMS the sensed data are collected and viewed in the local server. When the data present only in the local server mean that system will be a ordinary data collection one. When we stop the environment monitoring with the MMS the human work will not be stop. Example one person will need to be present in front of the server system and take the corresponding tasks from there. So here also we cant overcome one of our main problem,so that we need to switch this application to the another stage which is called as Remote Monitoring purpose for that we introduce the windows Azure technology in the MMS. For this RMMS system we may use many technologies but why we choose the Windows Azure for our system is because we develop the MMS application with the help of Visual Studio Dot-net which is a Microsoft product similarly Windows Azure also a Microsoft product so that we can easily interrogate both the things. And also the Windows Azure is able to support the SQL database. In our MMS system also we use the SQL so the the database conversion also not necessary here. In the RMMS system also the users need to login to access the data. After the user enter the RMMS he can view the sensed data values in the remote area. 


\section{International Journal of Science and Research (IJSR) \\ ISSN (Online): 2319-7064}

Index Copernicus Value (2013): 6.14 | Impact Factor (2015): 6.391

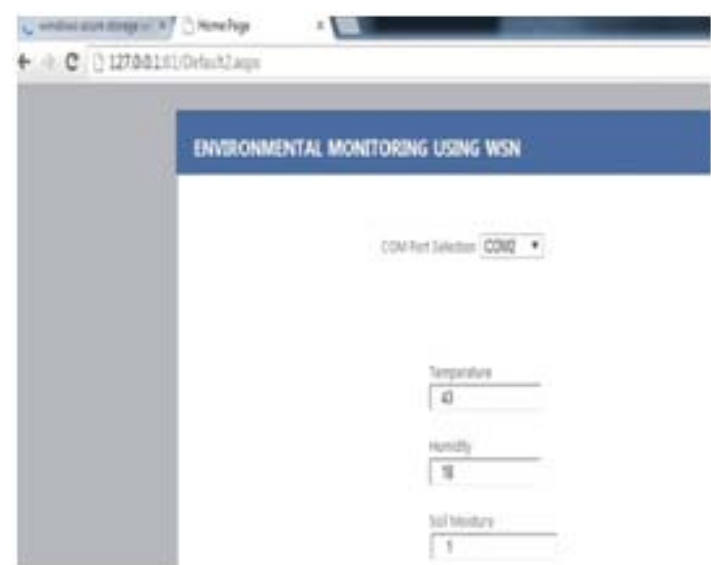

Figure 4: RMMS Application

We not stop our application with that because he know the environment changes of the agriculture area only when he logged into the system, but some formers may not know to access the Internet and see the change. For them we add the another feature in our RMMS. That is call Alert System. In that when the former going to use the the Sensors in there area we store there mobile number in that system with the help of micro controller.

Temperature level error!!
Value = 41 'CFor more info Check
Website
Humidity level error!!
Value = $81 \%$ For more info Check
Website
Soil Moiture level error!!
Value = 4For more info Check Website
Temperature level High!!!
Value = 41 'C
For more info Check Website
Mar $6,305 p M$ via sus
Humidity level High!!
Value = $84 \%$
For more info Check Website
Mar $6,3: 11$ pM via sus
Temperature level High!!!
Value = 41 'C
For more info Check Website

Figure 5: SMS Alert system

And we add the GSM interface kit with the micro controller with this system we can send the current climate status to the former directly for that we follow many conditions.

\begin{tabular}{|c|c|}
\hline S.No & \multicolumn{1}{c|}{ Conditions } \\
\hline 1 & $\begin{array}{r}\text { Every day the system transfer that day overall } \\
\text { environment condition }\end{array}$ \\
\hline 2 & $\begin{array}{c}\text { Based on the environment changes the system } \\
\text { transfer the message(eg: when the soil moister } \\
\text { content is very high we need to stop the water,so on } \\
\text { that time the alert message is transfer) }\end{array}$ \\
\hline 3 & $\begin{array}{c}\text { When the former register the system based on there } \\
\text { requirement the system send the alert to the formers }\end{array}$ \\
\hline
\end{tabular}

Figure 6: SMS Alert Condition

The above figure show about the Alert message which is transfer to the formers mobile.

\section{Conclusion and Future Enhancement}

Sensor networks have two significant differences from traditional networks, namely nodes have limited processing capability and power and a sensor network may consist of a huge number of nodes. As a result, the traditional layer architecture may not be appropriate since it is designed to support generality, rather than simplicity. As a result, a new architecture is provided which will help for remote monitoring of sensed parameter such as Temperature, Humidity and Soil Moisture. If sensed value exceeds the threshold level, message alerts are enabled and forwarded to the user using GSM interface kit, so the user can accordingly provide feedback control to the reference system to their requirement. C sharp is used for implementation and Window Azure platform is used as an emulator and using .NET Framework as front end and back end as SQL the results are taken and tabulated. The system provides better micro climate monitoring and control parameter such as temperature, humidity and soil moisture where controlled in an effective and efficient way. The present system can be expanded by addressing other micro climate parameter such as $\mathrm{PH}$ value, wind energy and salinity etc. A remote control interface can also be used for better facilitation of the devices in remote environment using $3 \mathrm{G}$ or $4 \mathrm{G}$ technology.

\section{References}

[1] Pahuja Roop, verma H.K and Uddin Moin,A wireless sensor network for greenhouse climate control Pervasive Computing.(volume 12,Is-sue:2),2013.

[2] Luca Bencini, Davide Di Palma, Giovanni Collodi and Gianfranco Manes,Wireless Sensor Systems for Onfield Agricultural Management Process,2010.

[3] J. Yick, B. Mukherjee, and D. Ghosal,Wireless sensor network sur-vey.Computer Networks, Vol. 52, Issue 12, pp. 2292-2330, August 2008.

[4] G. Barrenetxea, F. Ingelrest, G. Schaefer, and M. Vetterli,The hitchhiker's guide to successful wireless sensor network deployments.Proceedings of the 6th ACM conference on Embedded network sensor systems, 2008, Raleigh, NC.

[5] R. Cardell-Oliver, M. Kranz, K. Smettem, and K. Mayer,A Reactive Soil Moisture Sensor Network: Design and Field Evaluation,International Journal of Distributed Sensor Networks, vol. 1, pp. 149-162, 2005.

[6] IF. Akyildiz, W. Su, Y. Sankarasubramaniam, and E. Cayirci,Wireless Sensor Systems: a survey,Computer Networks; vol. 38, issue 4, pp. 393422, 2002.

[7] M. Beraka, H. Mathkour, S. Gannouni, H. Hashimi,Applications of Different Web Service Composition Standards,IEEE International Con-ference on Cloud and Service Computing (CSC), pp.56-63, Nov.2012.

[8] Cholatip Yawut and Sathapath KilasoA Wireless Sensor Network for Weather and Disaster Alarm System,International Conference on Infor-mation and Electronics Engineering,IPCSIT vol.6,2011.

[9] Peng Zhang,Zheng Yan and Hanlin SunA Novel Architecture Based on Cloud Computing for Wireless Sensor NetworkICCSEE,2013. 